\title{
Swarm in a fly bottle: Feedback-based Analysis of Self-organizing Temporary Lock-ins
}

\author{
Heiko Hamann ${ }^{1}$ and Gabriele Valentini ${ }^{2}$ \\ ${ }^{1}$ Department of Computer Science, \\ University of Paderborn, Paderborn, Germany \\ ${ }^{2}$ IRIDIA, Université Libre de Bruxelles, Brussels, Belgium \\ heiko.hamann@uni-paderborn.de, gvalenti@ulb.ac.be
}

May 20, 2014

\begin{abstract}
Self-organizing systems that show processes of pattern formation rely on positive feedback. Especially in swarm systems, positive feedback builds up in a transient phase until maximal positive feedback is reached and the system converges. We investigate alignment in locusts as an example of swarm systems showing time-variant positive feedback. We identify an influencing bias in the spatial distribution of agents compared to a well-mixed distribution and two features, percentage of aligned swarm members and neighborhood size, that allow to model the time variance of feedbacks. We report an urn model that is capable of qualitatively representing all these relevant features. The increase of neighborhood sizes over time enables the swarm to lock in a highly aligned state but also allows for infrequent switching between lock-in states.
\end{abstract}

\section{Introduction}

Many systems showing pattern formation, such as animal coloration [Camazine et al., 2001], embryogenesis [Crick, 1970], and grazing systems [Noy-Meir, 1975], are examples of self-organizing systems. In addition to multiple interactions of sub-components, general features of self-organizing systems are the interplay between positive feedback (also amplification or activation) and random fluctuations as well as that between positive and negative feedback (also inhibition) [Bonabeau et al., 1999]. Typically the system is initialized to an unordered state (not showing any patterns). Fluctuations generate deviations which are amplified by positive feedback until a spatiotemporal pattern forms. Negative feedback might prevent the system from reaching extreme states (e.g., 100\% ordered, extinction). Following this stochastic process, a random dynamical attractor forms and characterizes the dynamics of the system [Arnold, 2003]. 
Swarm systems are an example of self-organizing systems. A frequent setting in the case of swarms is that several stable ordered states exist (multistability) that are symmetrical to each other - a typical situation in a collective decisionmaking system. A swarm is a distributed agent system where each agent autonomously decides on its actions. With the global knowledge of an external observer, we can classify at least a subset of these actions as positive or negative feedback events that drive the system, respectively, towards or away from a too ordered state [Hamann, 2012, 2013b,a]. By counting these events we are able to calculate the ratio of positive feedback events $f^{+}=\frac{F^{+}}{F^{+}+F^{-}}$, for the number of positive feedback events $0 \leq F^{+} \leq N$ for swarm size $N$ ( $F^{-}$accordingly). If $f^{+}>0.5$ we say positive feedback is predominant. For several swarm systems, such as density classification, aggregation controlled by BEECLUST, and alignment in locust swarms, negative feedback is initially predominant while positive feedback builds up only over time and independently of the order of the current system state [Hamann, 2012, 2013b,a]. Consequently, there exists a second feature and/or a mechanism besides the order of the system that controls the increase of positive feedback intensity. This feature is very likely a spatial feature which can be determined by the method of elimination due to the simplicity of the investigated systems (agents have only 2 properties: direction of motion and position).

Our main objective is to determine the above mentioned second feature and to define an appropriate model that covers the interplay between positive and negative feedback as well as the increase of positive feedback over time. We continue the work reported in earlier publications [Hamann, 2012, 2013a] by focusing on questions raised therein. All of the following experiments are based on a swarm model inspired by swarm alignment of locusts. These swarms switch between different aligned states even after having reached high degrees of alignment. Such a special property is also subject to the following investigations.

\subsection{Locust scenario}

The desert locust, Schistocerca gregaria, shows collective motion in the growth stage of a wingless nymph often called 'marching bands' [Buhl et al., 2006]. The collective motion is expressed in the directional alignment of a majority of locusts, it is density-dependent, and individuals seem to change their direction as a response to neighbors [Buhl et al., 2006]. In experiments, the complexity of the collective motion is reduced to a pseudo-1-d setting by using a ring-shaped arena. Microscopic [Czirók et al., 1999] and macroscopic models [Yates et al., 2009, Hamann, 2012, 2013b,a] of this behavior have been reported. Here we use the microscopic model of self-propelled particles by Czirók et al. [1999] as our reference model (henceforth 'Czirók model'). The system is defined in 1d space. A particle $i$ has coordinate $x_{i} \in[0, C)$ and discrete, dimensionless velocity $u_{i} \in[-1,1]$. We refer to particles with velocity $u_{i}<0$ as 'left-goers' (respectively, 'right-goers' for $u_{i}>0$ ). The dynamics of a particle is defined by $x_{i}(t+1)=x_{i}(t)+v_{0} u_{i}(t)$ where $v_{0}$ is the nominal particle velocity and $u_{i}(t+$ 
1) $=G\left(\langle u(t)\rangle_{i}\right)+\xi_{i}$ considers its interaction with neighbors (subject to noise $\xi_{i}$ uniformly distributed over $[-\eta / 2, \eta / 2])$. The local average velocity $\langle u(t)\rangle_{i}$ for the $i$ th particle is calculated over all neighbors located in the interval $\left[x_{i}-\right.$ $\left.\Delta r, x_{i}+\Delta r\right]$ for perception range $\Delta r$ (see Table 1 for the parameter settings). $G$ describes both propulsion and friction forces

$$
G(u)=\left\{\begin{array}{ll}
(u+1) / 2, & \text { for } u>0 \\
(u-1) / 2, & \text { for } u<0
\end{array} .\right.
$$

The initial condition is a random uniform distribution for both the particles' coordinates $x_{i} \in[0, C)$ and their velocities $u_{i} \in[-1,1]$.

In the locust system, the spatial distribution of particles is biased and undergoes a nontrivial evolution. Fig. 2a gives a simplified picture of the spatial correlations generated by the Czirók model in the form of the pair correlation function. For a given left-goer ratio, we measure the density of left-goers as a function of the distance from a left-goer at times $t_{1}=30$ and $t_{2}=90$. We consider swarms with $N=41$ particles and system states with 25 left-goers and 16 right-goers only. The shown results are averaged over many independent runs. The two horizontal dashed lines give the expected distribution under the assumption of a uniform distribution of particles. Early in the simulation, at $t_{1}=30$, a left-goer has an increased density for nearby left-goers (within distances of about 2.6) in comparison to an assumed uniform distribution. Accordingly, right-goers have a decreased density for nearby right-goers due to symmetry. Later in the simulation, at $t_{2}=90$, left-goers have an increased density of nearby left-goers for even longer distances of up to about 6.0 and as a consequence a decreased density for the remaining arena (accordingly for right-goers). These spatial correlations in the particle distributions are discussed next and at the end of the paper we also interpret the temporal evolution of these correlations.

\section{Models}

We give a model to investigate the influence of biased spatial distributions as indicated above by the pair correlation function. A Markov chain model for two system variables is introduced to model the above mentioned second feature of the system, for which we choose the neighborhood size. We present an urn model that is able to represent the relevant spatial features and a mathematical model of the underlying feedback processes.

\subsection{Well-mixed and biased spatial distributions}

We model the collective decision-making process using Markov chains. A simple model for collective decision with only one state variable was reported before [Hamann, 2012, 2013a]. In the locust scenario, we count left-goers $L$ (without loss of generality) and get a set of $N+1$ states: $\{0,1, \ldots, N\}$. As simplifying assumptions, we ignore that the system might stay within the current 


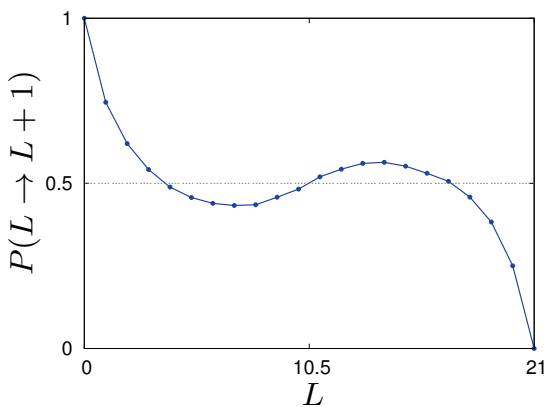

(a) Czirók model, transition probabilities

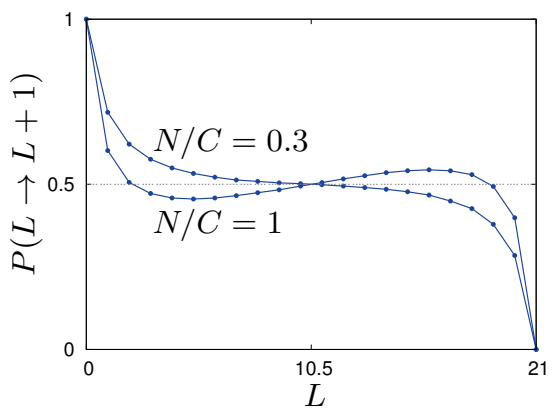

(c) well-mixed model, transition probabilities for different agent densities

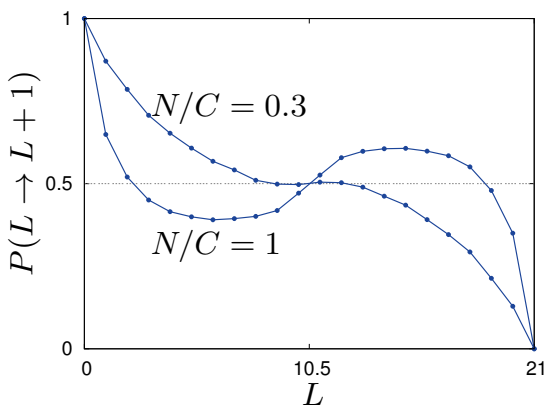

(e) bias model, transition probabilities

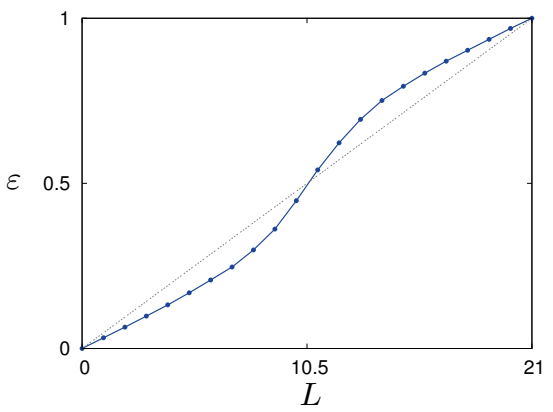

(b) Czirók model, left-goer edge ratio

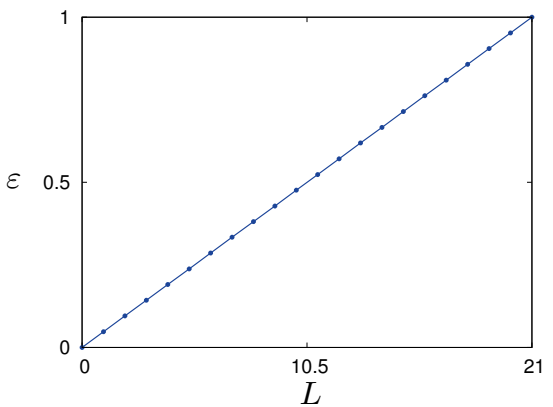

(d) well-mixed model, left-goer edge ratio

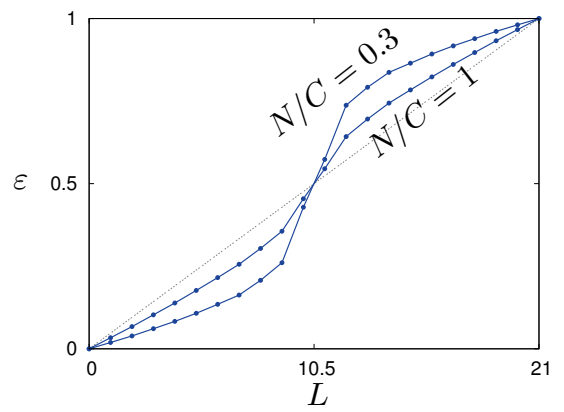

(f) bias model, left-goer edge ratio

Figure 1: Transition probabilities $P(L \rightarrow L+1)$ and left-goer edge ratio $\varepsilon$ using the Czirók model and two methods of initially positioning agents following a random uniform distribution or a special biased distribution $(N=21)$. 
state (i.e., no self-loops) and that we might have changes in the left-goer number of more than one particle within a small time interval. Without loss of generality, we focus exclusively on transitions that are increasing the number of left-goers $P(L \rightarrow L+1)$ due to the symmetry $P(L \rightarrow L+1)=1-P(L \rightarrow L-1)$ for $L \in\{1,2, \ldots, N-1\}$. These transition probabilities are measured using the Czirók model (see Fig. 1a).

An abstract model that only counts left-goers is not representing space, and therefore, implicitly assumes for the agents a well-mixed distribution in the space independent of their internal state (e.g., heading, opinion). However, swarm systems typically rely on spatial features and show non-homogeneous distributions of agents [Hamann, 2010]. In the locust scenario, the first priority for the swarm is to achieve alignment which is generally independent of agents' positions. However, locusts seem to depend heavily on spatial features such as the number of neighbors [Hamann, 2013b]. In the following, we briefly investigate the difference between well-mixed systems and systems whose agents' spatial distributions are biased by agents' headings.

For the following experiment, we initially place the agents by sampling from a uniform distribution and calculate the updates in agent directions $u_{i}$ according to the Czirók model. We simulate only one time step, and consequently, agents' positions are not correlated due to earlier dynamics. Fig. 1c shows the resulting transition probabilities for two agent densities $(N / C \in\{0.3,1.0\})$ based on $2 \times 10^{5}$ samples each. Agents' density influences the transition probabilities considerably. In addition, we note qualitative differences in the shapes of the curves compared to Fig. 1a.

Next, we define a measure capable to represent an important spatial feature. The spatial distribution of agents induces a graph. The existence of an edge is simply determined by checking whether two agents are mutually within their perception range $\Delta r$. The set of agents with whom an agent shares an edge defines also its neighborhood and the size of this set is its neighborhood size. To define our measure we count 'left-goer edges' which are edges that contain at least one left-goer. The set of left-goer edges $E_{L}$ is defined by $E_{L}=\{e \mid e=$ $\left(e_{1}, e_{2}\right)$ with $e_{1}$ and/or $e_{2}$ is left-goer $\}$. The definition of the set of right-goer edges $E_{r}$ is symmetrical. Our measure is the left-goer-edge ratio $\varepsilon$ which is calculated based on the set sizes: $\varepsilon=\left|E_{L}\right| /\left(\left|E_{L}\right|+\left|E_{R}\right|\right)$. The edge ratio $\varepsilon \in$ $[0,1]$ can be interpreted as an indicator of how the neighborhood sizes (i.e., node degrees) are distributed between left-goers and right-goers. If the neighborhood size averaged over all left-goers equals that averaged over all right-goers, then we have edge ratio $\varepsilon=0.5$. If the average neighborhood size of left-goers is bigger than that of right-goers, then we have edge ratio $\varepsilon>0.5$. If right-goer neighborhoods are bigger, then $\varepsilon<0.5$. We measure the edge ratio as a function of time for the Czirók model by averaging over $2 \times 10^{5}$ samples. Fig. 1 b shows the presence of bigger neighborhood sizes for left-goers in case of a global majority of left-goers $(L>N / 2$, for swarm size $N)$ and of smaller neighborhood sizes when left-goers are outnumbered $(L<N / 2)$. For the well-mixed simulation we do the same measurements as shown in Fig. 1d. For both densities the edge ratio scales linearly $\varepsilon(L)=L / N$ as expected due to the unbiased well-mixed distribution of 
agent positions. Hence, when well-mixed distributions are assumed, this spatial features of the Czirók model are ignored.

Next, we consider how the well-mixed simulation can be modified to introduce a spatial bias that results in a non-linear edge ratio $\varepsilon(L)$. A simple constructive approach to influence the edge ratio is to position the agents of the current majority in clusters of two. To create clusters of two, we position two agents from the majority group at the exact same position. Agents are initially positioned according to this procedure and their directions $u_{i}$ are updated for one time step. Averaging over many samples gives the resulting transition probabilities and edge ratio which are shown in Fig. $1 \mathrm{e}$ and $\mathrm{f}$ for two densities $(N / C \in\{0.3,1.0\})$. The biased positioning of agents influences both the edge ratio and transition probabilities. The increased density from 0.3 to 1.0 almost only introduces a downscaling of the edge-bias by a factor of about 0.45 .

\subsection{Markov chain model for two system variables}

We extend the Markov chain model reported before [Hamann, 2012, 2013a] by considering as second state variable the average neighborhood size $\mathcal{N} \in$ $\{1, \ldots, N\}$ over all agents (with perception range $\Delta r$ ). Fig. $2 \mathrm{~b}$ shows an example of the resulting chain for swarm size $N=3$. We get $(N+1) N$ states for swarm size $N$. For simplicity, we ignore again that the system might stay within the current state, that a concurrent change of both features might occur, and we also ignore that we might have changes in the left-goer number or neighborhood size of more than one within a small time interval. For any given state $(L, \mathcal{N})$, we measure the probability of observing a transition that increases/decreases the number of left- goers, $P(L \rightarrow L \pm 1 \mid(L, \mathcal{N}))$, and the probability of observing a transition that increases/decreases the neighborhood size, $P(\mathcal{N} \rightarrow \mathcal{N} \pm 1 \mid(L, \mathcal{N}))$. In this way we obtain a periodic Markov chain of period two due to the absence of self-loops in the chain. This kind of Markov chains does not converge to one stationary distribution but jumps between two stationary distributions: one for odd time steps and one for even time steps. We calculate a unique limiting distribution of the process by taking the mean of the two steady-state distributions of the chain. The analysis presented at the end of Sec. 3 is based on the computation of such limiting distributions.

\section{$2.3 \quad$ Urn model}

We define an urn model that represents most of the relevant features of the locust system, especially those that are due to spatial biases. Our aim is to develop a model that is simpler and faster to simulate than the Czirók model but still represents the qualitative key features of this system. The urn model consists of 3 urns: main, edges, and resource. Urn main represents the number of leftand right-goers in the swarm and contains a constant number of $N$ marbles. Urn edges represents an average neighborhood and contains a variable number of marbles $E$ which represents the neighborhood size and the edge ratio. Urn resource provides additional marbles to increase the neighborhood size $E$ and 
therefore also holds a variable number of marbles. At each round, the drawing process follows four stochastic rules (see Table 2 for used parameters).

Rule 1. We draw $E$ times from edges with replacement (if $E$ is even we do $E+1$ draws to avoid treatment of tie-breakers) and count the left-goers $\lambda$ and right-goers $\rho$ that we draw. Next, we draw one marble from main. If $\lambda>\rho$ and we have drawn a right-goer from main, then we put a left-goer back to main. If $\lambda<\rho$ and we have drawn a left-goer from main, then we put a right-goer back to main. Otherwise, that is, $\lambda<\rho$ and we have drawn a right-goer, we do not exchange the marble and put it back in main (accordingly for $\lambda>\rho$ and left-goer). This first drawing rule represents the actual decision process of an agent based on counting neighboring agents and a majority rule.

Rule 2. This second drawing rule is executed at each round only with a probability of $P_{\text {nsize. }}$. We draw $E$ times from edges with replacement (if $E$ is even we do $E+1$ draws) and count the left-goers $\lambda$ and right-goers $\rho$ that we draw. Next, we do $\min (\lambda, \rho)+1$ random experiments: with probability $P_{\text {incr }}$ we move a left-goer or a right-goer (with equal probability) from resource to edges if possible. Finally we do $E\left(\max (\lambda, \rho) / N-c_{\text {decr }}\right)$ random experiments and move with probability $P_{\text {decr }}$ a left-goer or a right-goer (with equal probability) from edges to resource if possible. This rule models the dynamics of the neighborhood size. Big neighborhoods increase their size faster than small neighborhoods for a balanced distribution of left- and right-goers. For unbalanced distributions, neighborhoods tend to decrease their size.

Rule 3. We draw one marble from main. If it is a left-goer, we replace a rightgoer in edges with a left-goer (if possible) or vice versa in the case of a right-goer (positive feedback). This third drawing rule is executed in each round only with a probability of $P_{\text {noise }}$.

Rule 4. We draw one marble from main. If it is a left-goer, we replace a left-goer in main with a right-goer (if possible) or vice versa in the case of a right-goer (negative feedback). This fourth drawing rule is executed in each round only with a probability of $P_{\text {noise }}$. These two last rules implement noise. They are executed with the same probability but the positive feedback operates on edges and negative feedback operates on main.

\subsection{Mathematical model of feedbacks}

We define a mathematical description of the above urn model. A detailed model would not allow for concise equations, therefore, we restrict our attention to the main features. We ignore the dynamics of urn edges except for the total number of marbles $E$ which gives the average neighborhoods size $\mathcal{N}$. Our main focus is to model the dynamics of urn main. We assume that the ratio of left-goers in the neighborhood (edges) is identical to the ratio of left-goers $m=L / N$ in main and that $E=\mathcal{N}$ is odd. For $\mathcal{N}$ marbles, of which $m \mathcal{N}$ are left-goers and $(1-m) \mathcal{N}$ are right-goers, the probability to draw a majority of left-goers is

$$
P_{\text {maj }}^{\text {left }}=\sum_{n \in\left\{\left\lceil\frac{\mathcal{N}}{2}\right\rceil, \ldots, \mathcal{N}\right\}}\left(\begin{array}{c}
\mathcal{N} \\
n
\end{array}\right) m^{n}(1-m)^{\mathcal{N}-n}
$$


and the probability to draw a majority of right-goers is

$$
P_{\text {maj }}^{\text {right }}=\sum_{n \in\left\{\left\lceil\frac{\mathcal{N}}{2}\right\rceil, \ldots, \mathcal{N}\right\}}\left(\begin{array}{c}
\mathcal{N} \\
n
\end{array}\right)(1-m)^{n} m^{\mathcal{N}-n} .
$$

Following a heuristic approach, the average change $\Delta m$ of left-goers within one time step is modeled as

$$
\Delta m^{h}(\mathcal{N}, m)=(1-m) P_{\mathrm{maj}}^{\mathrm{left}}-m P_{\mathrm{maj}}^{\mathrm{right}}-P_{\mathrm{noise}}(2 m-1),
$$

whereas the first two terms model the positive feedback effect implemented by rule 1. An increases (respectively, decrease) in the number of left-goers results from drawing a right-goer while having a majority of left-goers. Besides, the third term models the negative feedback effect of rule 4 .

As a second alternative, we model the average change $\Delta m$ of left-goers with a feedback-based approach as reported in [Hamann, 2012, 2013a]. That is, we neglect the actual processes causing positive and negative feedback in the the urn model and we focus instead on the probability of positive feedback $P_{F B}(\mathcal{N}, m)$. We get

$$
\Delta m^{F B}(\mathcal{N}, m)=1-2\left(\left(1-P_{F B}(\mathcal{N}, m)\right) P_{\text {maj }}^{\text {left }}+P_{F B}(\mathcal{N}, m) P_{\text {maj }}^{\text {right }}\right) .
$$

The probability of positive feedback is calculated by equating and solving the right hand sides of eqs. 4 and 5 which yields

$$
P_{F B}(\mathcal{N}, m)=-\left(2 m-15 P_{\mathrm{maj}}^{\text {left }}+5 m P_{\mathrm{maj}}^{\text {left }}+5 m P_{\mathrm{maj}}^{\mathrm{right}}+4\right) /\left(10 P_{\mathrm{maj}}^{\text {left }}-10 P_{\mathrm{maj}}^{\mathrm{right}}\right) .
$$

With increasing $\mathcal{N}$, we obtain polynomials of increasing degree. The first 3 are: $P_{F B}(\mathcal{N}=1, m)=2 / 5, P_{F B}(\mathcal{N}=3, m)=3 / 4-7 /\left(20\left(-2 m^{2}+2 m+1\right)\right)$ and $P_{F B}(\mathcal{N}=5, m)=3 / 4-7 /\left(20\left(6 m^{4}-12 m^{3}+4 m^{2}+2 m+1\right)\right)$. Fig. 2c shows the behavior of eq. 6 when $\mathcal{N} \in\{1,3, \ldots, 27\}$. A 'negative exponential' increase of positive feedback intensity with increasing neighborhood size $\mathcal{N}$ is clearly visible. A similar result was reported in [Hamann, 2013a, Fig. 8b] for a different swarm experiment showing temporal dependency. In the locust scenario, the neighborhood size also increases over time (see Section 3). Hence, the model given by eq. 6 indirectly confirms the increase of positive feedback in swarm systems as reported in [Hamann, 2012, 2013a].

\section{Results}

We investigate the Czirók model and the urn model with focus on the key findings that the average neighborhood size and the edge ratio are relevant features of the locust scenario. In particular, we investigate measured transition probabilities by interpreting both models as Markov chains (Sec. 2.2). An overview of the complete system dynamics is given by vector fields in Figs. $3 \mathrm{a}$ and $\mathrm{b}$ for $N=41\left(10^{6}\right.$ samples for Czirók model and $5 \times 10^{6}$ for urn model). These plots 


\begin{tabular}{|r|c|c|}
\hline parameter & sym. & value \\
\hline swarm size & $N$ & $\{17,21,25,33$, \\
& & $41,49,57,61\}$ \\
circumference & $C$ & $70(21)$ \\
nominal speed & $v$ & 0.1 \\
perception range & $\Delta r$ & 1.0 \\
noise & $\eta$ & 2.5 \\
\hline
\end{tabular}

\begin{tabular}{|r|c|c|}
\hline parameter & symbol & value \\
\hline prob. neighbh. rule (rule 2) & $P_{\text {nsize }}$ & 0.2 \\
prob. neighbh. size increase & $P_{\text {incr }}$ & 0.18 \\
prob. neighbh. size decrease & $P_{\text {decr }}$ & 0.007 \\
offset neighbh. size decrease & $c_{\text {decr }}$ & 0.15 \\
probability of noise & $P_{\text {noise }}$ & 0.2 \\
\hline
\end{tabular}

Table 1: Used parameters for the Czirók model.

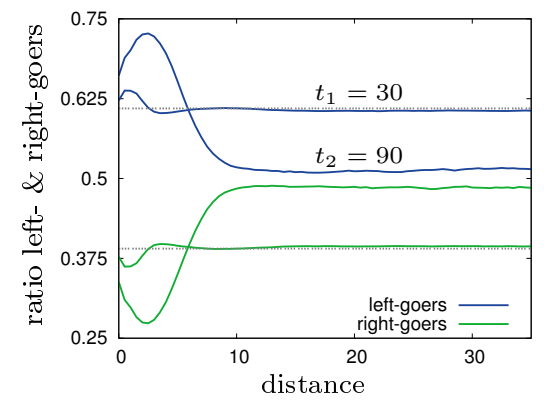

(a) Pair correlation function: measured density of left-/right-goers at distances from a particle of the same kind.

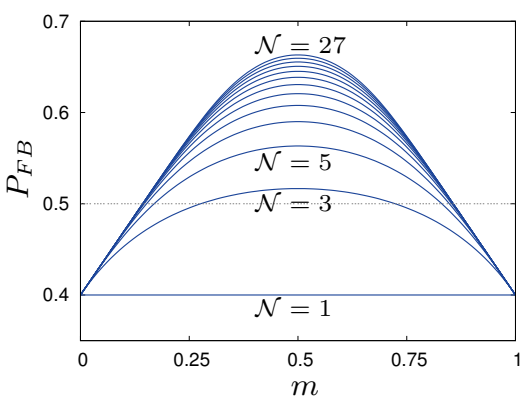

(c) Analytically obtained probabilities of positive feedback (eq. 6) for neighborhood sizes $\mathcal{N} \in\{1,3, \ldots, 27\}$.
Table 2: Used parameters for the urn model.

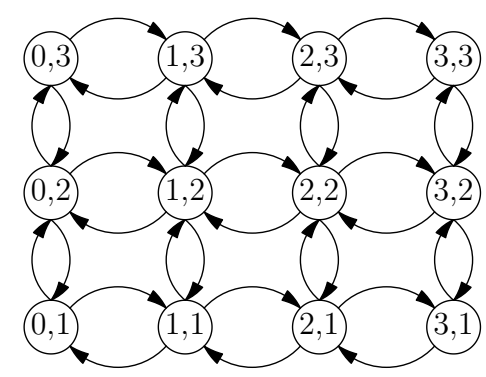

(b) Markov chain for two state variables: number of left-goers $L$ and the average neighborhood size $\mathcal{N}$ (swarm size $N=3$ ).

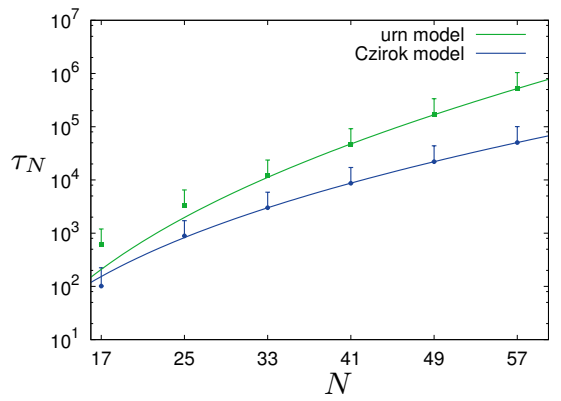

(d) Mean first passage time $\tau_{N}$ over swarm size $N$ for both models fitted to $a \exp (c x) x^{b}$, error bars give standard deviation.

Figure 2: Pair correlation, Markov chain, probability of feedback and mean first passage time.

are based on the transition probabilities which are put in relation to each other. Furthermore, the horizontal and the vertical lengths of the arrows were normalized individually to maximize readability (i.e., vector field plots are qualitative, the quantitative data is given in Figs. 3c-f). In the case of the Czirók model, as a consequence of the initial random uniform distribution of agents over the whole ring, the neighborhood size $\mathcal{N}$ is initially small $(2 \Delta r N / C \approx 1.2)$. Similarly, urn edges initially holds one left-goer and one right-goer. In the initial unordered 


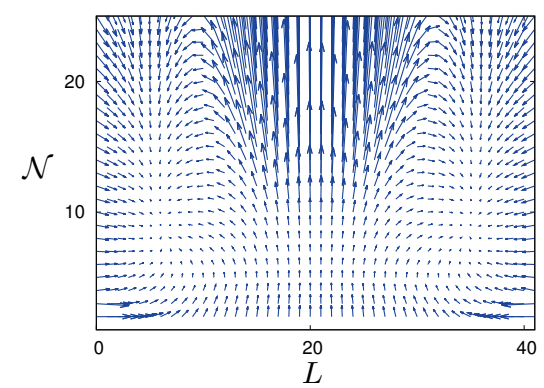

(a) Czirók model, vector field

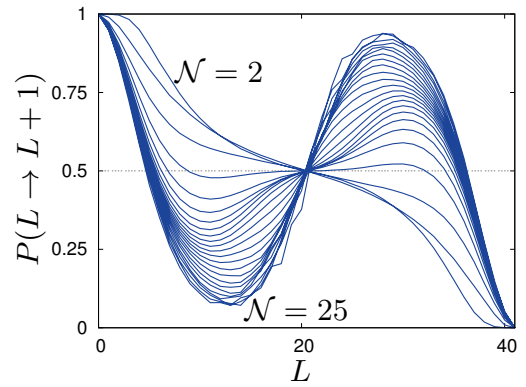

(c) Czirók model, transition probabilities number of left-goers $L$ (alignment)

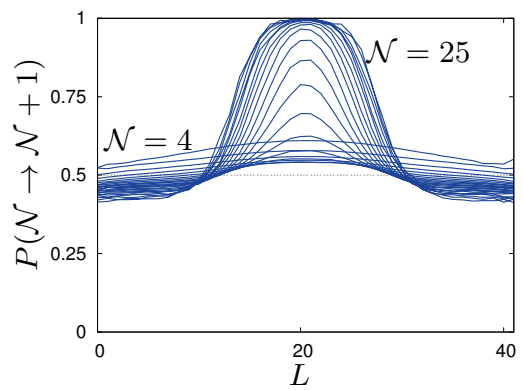

(e) Czirók model, transition probabilities neighborhood size $\mathcal{N}$

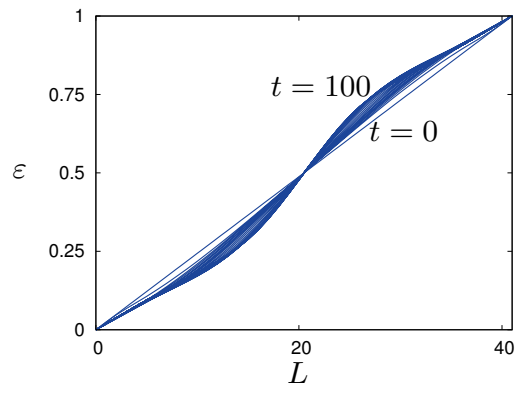

(g) Czirók model, left-goer edge ratio $\varepsilon$

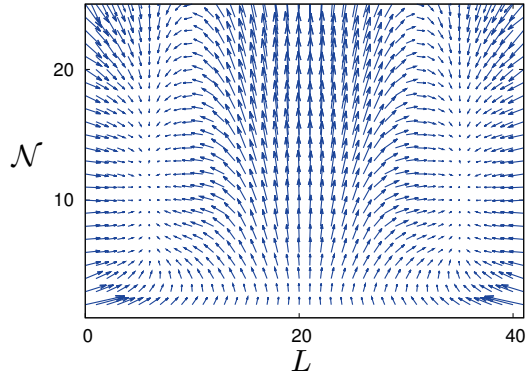

(b) urn model, vector field

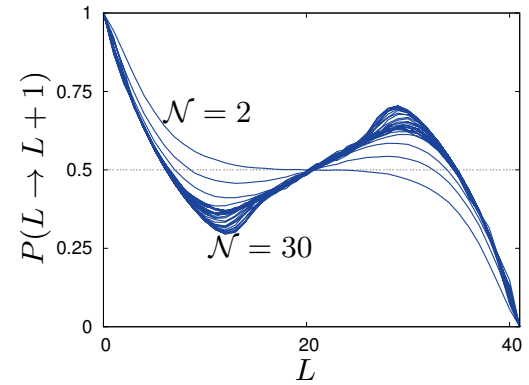

(d) urn model, transition probabilities number of left-goers $L$ (alignment)

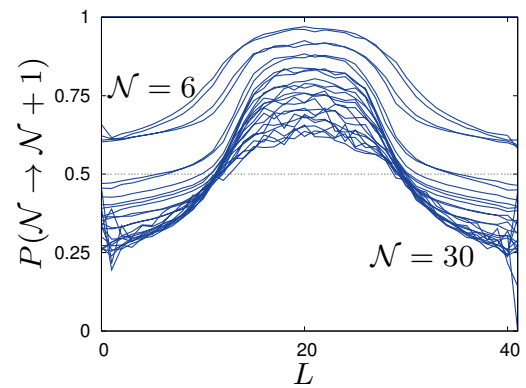

(f) urn model, transition probabilities neighborhood size $\mathcal{N}$

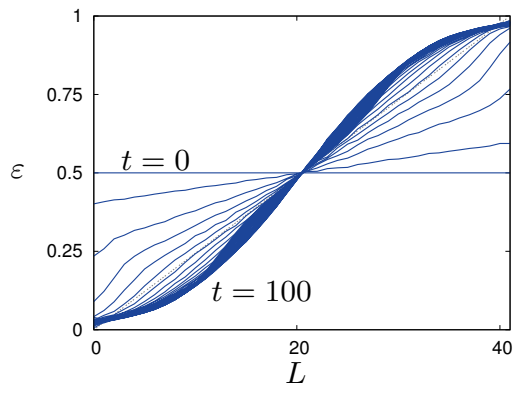

(h) urn model, left-goer edge ratio $\varepsilon$

Figure 3: Vector fields, transition probabilities, and edge ratio for the Czirók and urn model $\left(N=41,10^{6}\right.$ samples for Czirók model and $5 \times 10^{6}$ for urn model). 
state there are approximately the same number of left-goers and right-goers $(L \approx N-L)$. Hence, both systems start in the area at the lower middle of the vector field. First, the neighborhood size increases. Only later, once a bigger neighborhood size is formed, the system either increases or decreases in the number of left-goers $L$ until reaching a stable state, respectively, $(L, \mathcal{N}) \approx(35,8)$ or $(L, \mathcal{N}) \approx(6,8)$.

Projections of the data given in Figs. 3a and b are given in diagrams c-f. Figs. 3c and d give the transition probabilities for an increase in $L$ for all $\mathcal{N}$ for Czirók and urn model and e and $\mathrm{f}$ give the transition probabilities for an increase in $\mathcal{N}$ for all $\mathcal{N}$. Some curves are noisy because the corresponding configurations occur very rarely. We ignore statistical significance within this qualitative study (big quantitative differences between the two models are obvious). Positive feedback is found within approximately the same intervals in Figs. 3e and $\mathrm{f}$ (similarly for negative feedback). Noticeable is the extreme positive feedback for $6<L<18$ and $23<L<35$ for the Czirók model seen in Figs. 3c and for $12<L<29$ in e. Figs. $3 \mathrm{~g}$ and $\mathrm{h}$ give the left-goer edge ratio $\varepsilon$ for different times. The edge ratio of the urn model is more dynamic because the urn model is always started with one left-goer and one right-goer, that is, an edge ratio of $\varepsilon=0.5$. The initial edge ratio for the Czirók model, in turn, is the direct result of the uniform distribution of agents which gives $\varepsilon(L)=L / N$.

Finally, we investigate the scalability of the mean switching time between stable modes of the system. We look at the mean time $\tau_{N}$ necessary to move from an initial set of states with a majority of right-goers to a final set of states where left-goers lead the system. We consider swarm systems whose transient dynamics have already vanished and thus settled down to their limiting behavior. We define the set of initial (respectively, final) states looking at the limiting distribution of the process (computed from the Markov chain defined in Sec. 2.2). We select states with a majority of right-goers (respectively, left-goers) in ascending order of probability up to an overall joint probability of the set of 0.1. This corresponds to a majority of right-goers where $L / N \approx 0.15$ and a majority of left-goers with $L / N \approx 0.85$, while $\mathcal{N} \in[5.8,14.2]$ for the urn model and $\mathcal{N} \in[4,10.6]$ for the Czirók model. To compute the mean switching time $\tau_{N}$, we first lump together all final states with majority of left-goers in a single state and then we make this state absorbing. The mean switching time of our original chain corresponds to the absorption time of a process in the modified chain that starts with an initial distribution proportional to the limiting probabilities of the initial states selected so far. Fig. $2 \mathrm{~d}$ shows the mean switching time $\tau_{N}$ when the swarm size $N$ increases. The qualitative behavior of the urn model is similar to that of the Czirók model for all swarm sizes, with the latter experiencing shorter switching times. For both models, the mean switching time scales approximately exponentially with the size of swarm thus showing that bigger swarms form more stable majorities. 


\section{Discussion and conclusion}

This paper started from the result of earlier publications [Hamann, 2012, 2013a] that, in swarm systems, positive feedback builds up in a transient phase independently of the order parameter (here $L$ ) until maximal positive feedback is reached. In turns, this indicates the existence of a second feature that controls the increase in positive feedback. We identify the average size of agents' neighborhoods as this second feature and, in addition, we detect the relevance of the edge ratio. We extended the Markov-chain approach introduced in [Hamann, 2012, 2013a] to model the second state variable and therefore to count both left-goers and average neighborhood size. Although it was necessary to consider the original spatial features of the locust scenario, we extended the urn model concept [Hamann, 2012, 2013a] to mimic spatiality, particularly, neighborhood size and edge ratio.

The vector fields depicted in Figs. 3a and b provide a clear picture of what we call the "fly-bottle effect" . The swarm system is initialized with $L / N \approx 0.5$ and $\mathcal{N}<5$. At first, there is no positive feedback concerning the number of left-goers $L$ (Figs. 3c and d) but the average neighborhood size $\mathcal{N}$ increases (in analogy to the fly bottle: 'entering from below', Figs. 3e and f). Once $\mathcal{N} \approx 10$ or bigger, a strong positive feedback emerges that easily breaks the symmetry given by $L / N \approx 0.5$ and drives the system towards $L / N \approx 0.12$ or $L / N \approx 0.88$ (fly-bottle analogy: phototaxis behavior). For these values, however, there is negative feedback on $\mathcal{N}$ which decreases to $\mathcal{N} \approx 8$. Finally, positive and negative feedbacks balance out and the system converges to either $L / N \approx 0.15$ or $L / N \approx$ 0.85 generating a lock-in effect ('the fly is trapped'). This lock in effect is only temporary because the positive feedback operating on $L$ for $\mathcal{N} \approx 8$ is much weaker than for $\mathcal{N}>8$. In the long run, the system shows a switching behavior between the two lock-ins. However, such a change in majority becomes more infrequent with increasing swarm size $N$ as also seen in natural locusts [Buhl et al., 2006]. The fly-bottle effect, that relies on a second feature serving as a kick starter for the whole system, seems to have a certain generality in swarm systems. Indeed, the increase of positive feedback during a transient phase was also reported for other systems such as density classification and aggregation controlled by BEECLUST [Hamann, 2012, 2013a].

An additional interpretation of the fly-bottle effect with reference to Fig. 2a is that the secondary feature is generating only short-ranged correlations early on but not global correlations. These short-ranged correlations seem to be sideeffects, such as small clusters of agents in the investigated locust scenario. The long-range correlations seen later in the system are then an effect of the primary feature (here $L$ ) and probably could neither be generated by the one or the other feature alone. Future research work will focus on the questions whether the flybottle effect is generally observed in swarm systems and, if so, whether it can be used to design swarm behaviors for artificial swarm systems.

\footnotetext{
${ }^{1} \mathrm{~A}$ fly bottle is a traditional device made of clear glass to passively trap flying insects that enter it from below and cannot escape because of their phototaxis behavior.
} 


\section{Acknowledgments.}

This work was partially supported by the European Research Council through the ERC Advanced Grant "E-SWARM: Engineering Swarm Intelligence Systems" (contract 246939). Thanks to Y. Khaluf for helpful comments.

\section{References}

Ludwig Arnold. Random Dynamical Systems. Springer, 2003.

Eric Bonabeau, Marco Dorigo, and Guy Theraulaz. Swarm Intelligence: From Natural to Artificial Systems. Oxford Univ. Press, New York, NY, 1999.

Jerome Buhl, David J. T. Sumpter, Iain D. Couzin, Joe J. Hale, Emma Despland, E. R. Miller, and Steve J. Simpson. From disorder to order in marching locusts. Science, 312 (5778):1402-1406, 2006. doi: 10.1126/science.1125142.

Scott Camazine, Jean-Louis Deneubourg, Nigel R. Franks, James Sneyd, Guy Theraulaz, and Eric Bonabeau. Self-Organizing Biological Systems. Princeton University Press, Princeton, NJ, 2001.

Francis Crick. Diffusion in embryogenesis. Nature, 225(5231):420-422, 1970. doi: 10.1038/ 225420a0. URL http://dx.doi.org/10.1038/225420a0.

András Czirók, Albert-László Barabási, and Tamás Vicsek. Collective motion of self-propelled particles: Kinetic phase transition in one dimension. Physical Review Letters, 82(1):209212,1999

Heiko Hamann. Space-Time Continuous Models of Swarm Robotics Systems: Supporting Global-to-Local Programming. Springer, Berlin, Germany, 2010.

Heiko Hamann. Towards swarm calculus: Universal properties of swarm performance and collective decisions. In Marco Dorigo, Mauro Birattari, Christian Blum, Anders Lyhne Christensen, Andries Petrus Engelbrecht, Roderich Groß, and Thomas Stützle, editors, Swarm Intelligence: 8th International Conference, ANTS 2012, volume 7461 of LNCS, pages 168-179, Berlin, Germany, 2012. Springer. URL http://dx.doi.org/10. 1007/978-3-642-32650-9_15.

Heiko Hamann. Towards swarm calculus: Urn models of collective decisions and universal properties of swarm performance. Swarm Intelligence, 7(2-3):145-172, 2013a. URL http: //dx.doi.org/10.1007/s11721-013-0080-0.

Heiko Hamann. A reductionist approach to hypothesis-catching for the analysis of selforganizing decision-making systems. In 7th IEEE Int. Conf. on Self-Adaptive and SelfOrganizing Systems (SASO 2013), pages 227-236, Los Alamitos, CA, 2013b. IEEE Press. doi: 10.1109/SASO.2013.10.

Imanuel Noy-Meir. Stability of grazing systems: an application of predator-prey graphs. The Journal of Ecology, 63(2):459-481, 1975.

Christian A. Yates, Radek Erban, Carlos Escudero, Iain D. Couzin, Jerome Buhl, Ioannis G. Kevrekidis, Philip K. Maini, and David J. T. Sumpter. Inherent noise can facilitate coherence in collective swarm motion. Proc. Natl. Acad. Sci. USA, 106(14):5464-5469, 2009. doi: 10.1073/pnas.0811195106. URL http://www.pnas.org/content/106/14/5464.abstract. 